\title{
Examining the Relationship Between Introductory Computing Course Experiences, Self-Efficacy, and Belonging Among First-Generation College Women
}

\author{
Jennifer M. Blaney \\ University of California, Los Angeles \\ Moore Hall, 457 Portola Plaza \\ Los Angeles, CA 90095 \\ jmblaney@ucla.edu
}

\author{
Jane G. Stout \\ Computing Research Association \\ 1828 L St. NW, Ste. 800 \\ Washington D.C., 20036 \\ jane@cra.org
}

\begin{abstract}
Computing self-efficacy and sense of belonging are known predictors of motivation and persistence. As such, these psychological states are important to study in order to broaden participation in computing. This study examined the relationship between (a) introductory computing course experiences and (b) self-efficacy and sense of belonging in computing, focusing on differences by gender and college generation status. We found that the relationship between some introductory course experiences and self-efficacy and sense of belonging was strongest among first-generation college women, which reveals the importance of considering women's experiences in light of their additional intersectional identities. Recommendations for best practices in introductory computing courses are discussed.
\end{abstract}

\section{Keywords}

Undergraduate students; first-generation students; gender; introductory computing courses; self-efficacy; sense of belonging

\section{INTRODUCTION}

Women's representation in computing has become a national focus in recent years. Despite this, efforts to broaden women's participation in computing have been met with limited success, as women remain underrepresented in the field, making up approximately $18 \%$ of computer science degrees awarded [10]. One reason for limited success in increasing women's representation in the field may be that little is known about women's intersectional experiences in computing, or the differential experiences of women as a function of their other identities (e.g., racial/ethnic identity, socioeconomic status, etc.). This paper specifically focuses on students' experiences at the intersection of gender and first-generation college status.

\subsection{Women First-Generation College Students: A Doubly Disadvantaged Group in Computing}

First-generation college students can be conceptualized as belonging to a disadvantaged group within academia. That is, relative to their counterparts (continuing generation students), first-generation students tend to (a) lack a strong support network within their family who can help guide students through college, and (b) come from lower socioeconomic backgrounds, resulting in fewer economic resources for college amenities (e.g., laptops) and

Permission to make digital or hard copies of all or part of this work for personal or classroom use is granted without fee provided that copies are not made or distributed for profit or commercial advantage and that copies bear this notice and the full citation on the first page. Copyrights for components of this work owned by others than ACM must be honored. Abstracting with credit is permitted. To copy otherwise, or republish, to post on servers or to redistribute to lists, requires prior specific permission and/or a fee. Request permissions from Permissions@acm.org.

SIGCSE'17, March 8-11, 2017, Seattle, WA, USA.

(C) 2017 ACM. ISBN 978-1-4503-4698-6/17/03...\$15.00.

DOI: http://dx.doi.org/10.1145/3017680.3017751 necessities (e.g., books), and a greater need to divide one's time by working at a job outside of college [12].

Within the computing community, women are a disadvantaged group, as they contend with negative stereotypes about their ability in computing and a chronic sense that they do not "belong" [15]. Together, existing research indicates that women who are also first-generation college students may constitute a doubly disadvantaged group in computing. Importantly, recent research on computer science students has found that first-generation students from underrepresented populations in computing (e.g., women) show a stronger relationship between sense of belonging and achievement in computing, compared to their peers [16]. This suggests that first-generation women studying computing may respond positively to inclusive course environments.

\subsection{Self-Efficacy and Belonging in Computing}

The current research observes the relationship between introductory computing course experiences and two important psychological states: self-efficacy and belonging in computing. A sense of belonging is defined as the subjective feeling of fitting in and being included as a valued and legitimate member of an academic discipline, and is a known predictor of academic persistence and achievement [3] [4] [19]. Self-efficacy refers to beliefs about one's ability to plan for and execute steps necessary for future success. Research has shown that self-efficacy promotes academic performance and motivation [8].

A great deal of prior research on computer science education has focused on women's recruitment and degree attainment, but less research has examined the quality of women's experiences in the classroom and other affective outcomes for women in computing that may ultimately lead to retention in the field (e.g., sense of belonging). This gap in the existing literature is particularly important given that degree attainment is only one measure of women's representation in computing. Furthermore, some research indicates that women are significantly more likely than their male counterparts to leave computing, even after earning a degree in the field [13]. Moreover, because so few women pursue computing as a career path, it is particularly important to understand how to best retain the small number of women who have opted into postsecondary coursework in computing. Therefore, in order to increase women's representation in computing during and after college, the current research examines psychological states that are precursors to retention in the field.

\subsection{Introductory Course Experiences}

The current study focused on three variables measuring common experiences with introductory computing courses that we expect to be positively related to self-efficacy and belonging for all students, but especially women who are first-generation college 
students: frequency of student-instructor interactions in the introductory course classroom; frequency of student-instructor interactions outside the classroom; and students' reports of the extent to which the instructor was inclusive to all students.

Prior research and theory has shown that interacting with faculty predicts a myriad of positive outcomes for students, including improved self-conceptions, academic success, degree aspirations, and retention [11] [2]. Furthermore, research has shown that student-faculty interactions may be particularly helpful in developing underrepresented students' sense of belonging and fit within STEM [6]. Related to the current work, interactions with faculty have been shown to be differentially impactful for different groups of students in a number of ways. As a case in point, working directly with faculty on research has been shown to be more impactful for women than for men in developing an interest in STEM research careers [14].

In addition to interpersonal interactions with instructors, this study examines the impact of instructor inclusivity on self-efficacy and belonging in computing. Instructor inclusivity is a measure of one aspect of classroom "climate" and includes students' reports of introductory course instructors' willingness to help students regardless of gender and race/ethnicity. Prior research and theory indicates that an inclusive classroom climate is predictive of students' sense of belonging and success in STEM fields [6].

\subsection{Study Overview}

This paper contributes to existing literature on women in computing by examining the relationship between introductory computing course experiences and both computing self-efficacy and sense of belonging, among women and men, with consideration of first-generation status. The following questions guide our inquiry:

1. Among introductory computing students, do self-efficacy and sense of belonging vary for first-generation women compared to other groups?

2. Do first-generation women have different levels of exposure to beneficial introductory course experiences compared to their peers?

3. To what extent are introductory course environments associated with self-efficacy and sense of belonging, and is this relationship different for first-generation women compared to their peers?

\section{DATA AND METHODS 2.1 Sample}

This study utilized data collected during fall of 2015 by the Computing Research Association's Center for Evaluating the Research Pipeline (CERP) and the Building, Recruiting, and Inclusion for Diversity (BRAID) research project. CERP and the BRAID research team have forged a collaborative data collection initiative that annually surveys undergraduate students enrolled in computing courses at a sample of institutions. For the purposes of this study, we focused on data collected from undergraduate students who were enrolled in an introductory computing course during fall of 2015 , resulting in $\mathrm{N}=2,184$ students who were enrolled at one of 65 universities across the United States.

The sample included responses from 704 women (83 firstgeneration to college students, 577 continuing generation students, 44 missing data points) and 1,345 men (195 firstgeneration college students, 1,050 continuing generation students, 100 missing data points). Gender was measured by students' selfreported identity as female, male, or non-binary. Students who indicated a non-binary gender identity were not included in our analysis due to small sample size. To determine generation status, students were asked to report the highest education level for each of their parents including: less than high school; high school or GED; some college; bachelor's degree; master's degree; $\mathrm{PhD}$; and professional degree. Students were counted as first-generation when their parent(s) had an education level of either a) less than high school or b) high school graduate or GED. If one or more parent had an education level of some college or higher, the student was considered continuing generation.

\subsection{Measures}

This study included three variables that are latent constructs: selfefficacy in computing, sense of belonging in computing, and perceived instructor inclusivity. Latent constructs refer to variables that cannot be directly measured or observed; thus, they are best measured through confirmatory factor analysis [9]. Confirmatory factor analysis (via principle axis factoring) was used to identify and compute variables to measure the three latent constructs. Through factor analysis, items were weighted (see factor loadings in the Appendix, Table A1) and added together to create factor scores, which were standardized and centered at the mean, which is equal to 0 .

\subsubsection{Self-Efficacy and Belonging Variables}

Self-efficacy in computing was measured by a factor made up of seven items asking students to report their confidence in their ability to: 1) find employment in their area of computing interest; 2) become a leader in the field of computing; 3) win a computingrelated contest; 4) get admitted to a graduate program in computing; 5) complete an undergraduate degree in computing; 6) quickly learn a new programming language; and 7) clearly communicate technical problems and solutions to a range of audiences, using a scale ranging from (1) strongly disagree to (5) strongly agree. This factor had a Cronbach's alpha of .89; the factor was standardized and mean-centered at zero, such that negative values indicate low self-efficacy, and positive values indicate high self-efficacy, compared to the grand mean (i.e., the mean of all students included in the sample). Factor loadings are included in the Appendix, Table A1, and descriptive statistics for this factor are included in the Appendix, Table A2.

Sense of belonging in computing was measured by a factor made up of three self-reported items (Cronbach's alpha = .74): I feel like I belong in computing; I feel like an outsider in the computing community (reverse-coded); and I feel welcomed in the computing community, using a scale ranging from (1) strongly disagree to (5) strongly agree. This factor was also standardized and meancentered at zero. See the Appendix, Tables A1 and A2 for factor loadings and descriptive statistics, respectively.

\subsubsection{Introductory Course Variables}

Frequency of interactions with instructors in the classroom was measured with a single item asking students to report how often they communicate with their introductory course instructor in class. This item was rated using the following scale: (1) never, (2) less than once per month, (3) one to three times per month, (4) one to three times per week, (5) more than three times per week.

Frequency of interactions with instructors outside of the classroom was measured using five items asking students to report the frequency that they interacted with instructors in the following ways: at office hours; by email; by phone call; by text message; and in informal meetings. Given high internal reliability (Cronbach's alpha of .81), we averaged these five items to create 
a composite score for data analysis. These items were rated using the following scale: (1) never, (2) less than once per month, (3) one to three times per month, (4) one to three times per week, (5) more than three times per week. Note that factor analysis was not needed to create the composite measure, as this measure of frequent instructor interactions is not a latent construct. As such, both measures of student-instructor interactions were kept on their original scaling so that 5 represents the greatest frequency.

Perceptions of instructor inclusivity were measured using a factor made up of five items asking students to rate their agreement with the following: introductory course faculty are inclusive and supportive of women; introductory course faculty are inclusive and supportive of students of color; introductory course faculty are interested in helping me when I come to them with questions; introductory course faculty are responsive to questions in class; and introductory course faculty are responsive to email communication, on a scale from (1) strongly disagree to (5) strongly agree. This factor had a Cronbach's alpha of .89 and was standardized and mean-centered at zero. Factor loadings are included in the Appendix, Table A1, and descriptive statistics for this measure are included in the Appendix, Table A2.

\section{FINDINGS}

\subsection{Differences in Self-Efficacy and Belonging}

Research question one asked: Among introductory computing students, do self-efficacy and sense of belonging vary for firstgeneration women compared to other groups? To address this research question, we assessed students' self-efficacy and sense of belonging as a function of their gender and generation status. Our analytic strategy was to run two separate one-way analyses of variance (ANOVAs), treating a four-level categorical variable of the four different demographic groups (i.e., first-generation women, continuing women, first-generation men, and continuing men) as between subjects factors. Self-efficacy and sense of belonging were treated as two separate outcome variables.

We found that groups reported different levels of self-efficacy, $F(3,1,886)=32.50, p<.001$, as well as belonging, $F(3,1,886)=$ $51.70, p<.001$. Post hoc Dunnett tests revealed that firstgeneration women reported significantly lower self-efficacy and belonging compared to their peers, each $p<.05$. Descriptive statistics for each of the four groups are included in Table 1.

Table 1. Self-Efficacy and Sense of Belonging as a function of Gender and Generation Status

\begin{tabular}{ccc}
\hline & Mean & Std. Error \\
\hline Computing Self-Efficacy & & \\
Gender/First-Generation & & \\
FG Women & -.52 & .11 \\
Continuing Women & $\mathbf{- . 2 6}$ & .01 \\
FG Men & $\mathbf{. 0 5}$ & .07 \\
Continuing Men & $\mathbf{. 1 7}$ & .03 \\
Sense of Belonging in Computing & & \\
Gender/First-Generation & & \\
FG Women & $\mathbf{- . 6 0}$ & .11 \\
Continuing Women & $\mathbf{- . 3 1}$ & .04 \\
FG Men & $\mathbf{. 0 8}$ & .07 \\
Continuing Men & $\mathbf{. 2 4}$ & .03 \\
\hline
\end{tabular}

Note. Both factors are standardized and mean-centered at zero, such that negative values represent lower self-efficacy and belonging, and positive values represent higher selfefficacy and belonging. Bold mean values indicate significantly different mean scores, compared against first-generation women, $p<.05$. FG = first-generation.

\subsection{Demographic Differences in Exposure to Introductory Course Experiences}

Research question 2 asked: Do first-generation women have different levels of exposure to beneficial introductory course experiences compared to their peers? To test this, we ran three separate one-way ANOVAs, again treating our four-level categorical demographic variable as between subjects factors, and (1) frequency of interactions with instructors in the classroom (2) frequency of interactions outside of the classroom, and (3) perceptions of inclusivity as outcome variables.

We found that students differed in their reported exposure to the three introductory course experiences of interest (frequency of faculty interaction in class, $F(3,1,862)=9.02, p<.001$; frequency of faculty interactions outside of class, $F(3,1,826)=$ $4.41, p<.001$; inclusivity, $F(3,1,844)=5.30, p<.001)$. Post hoc Dunnett tests did not reveal different levels of exposure for first-generation women, with the following exception: firstgeneration women reported less frequent interactions with faculty during class than continuing generation men, $p<.05$. Because we did not predict any other pattern of effects for the three introductory course experiences, we did not run additional post hoc tests. See Table 2 for descriptive statistics for each of the three introductory course variables by gender and generation status.

Table 2. Exposure to Introductory Course Experiences as a function of Gender and Generation Status

\begin{tabular}{lcc}
\hline & Mean & Std. Error \\
\hline $\begin{array}{l}\text { Frequency of faculty interaction in } \\
\text { class }\end{array}$ & & \\
Gender/First-Generation & & \\
FG Women & 2.58 & .05 \\
Continuing Women & 2.42 & .15 \\
FG Men & $\mathbf{2 . 8 8}$ & .04 \\
Continuing Men & 2.64 & .09 \\
Frequency of faculty interactions & & \\
outside of class & & \\
Gender/First-Generation & 1.44 & .02 \\
FG Women & 1.51 & .09 \\
Continuing Women & 1.51 & .02 \\
FG Men & 1.62 & .05 \\
Continuing Men & & \\
$\quad$ Inclusivity & & \\
Gender/First-Generation & .03 & .04 \\
FG Women & -.10 & .11 \\
Continuing Women & .08 & .03 \\
FG Men & -.22 & .08 \\
\hline Continuing Men &
\end{tabular}

Note. The faculty interaction variables are reported in their original metric, where 1 is the minimum frequency and 5 is the maximum frequency. The inclusivity factor is standardized and mean-centered at zero, such that negative values represent lower perceived inclusivity and positive values represent higher perceived inclusivity. Bold mean values indicate significant differences from first-generation women, $p<.05$. FG = first-generation.

\subsection{The Relationship Between Intro Course Experiences and Self-Efficacy/Belonging for Each Demographic Group}

Research question 3 asked: To what extent are introductory course environments associated with self-efficacy and sense of belonging, and is this relationship different for first-generation 
women compared to their peers? To address this question, we examined the relationship between introductory course experiences and self-efficacy and belonging via Pearson correlation tests. We first observed correlations for the full sample (preliminary analysis). Then, we observed correlations among the four student groups (primary analysis). In the correlation analyses that follow, we use Cohen's (1988) guidelines for interpreting the magnitude of correlations: $.10-.29$ is a small effect; $.30-.49$ is a medium effect; and .50 or greater is a large effect. Values less than .10 are considered inconsequential, and are not discussed.

Preliminary correlation analysis. We first observed the relationship between all three introductory course variables and self-efficacy and belonging for our full sample (see Table 3). All three introductory course experiences were positively associated with students' self-efficacy in computing, but the magnitude of each correlation was small. Frequent faculty interactions in class and inclusivity were also positively associated with sense of belonging (these effects were small), but frequency of faculty interactions outside of class was not (see Table 3 for correlation coefficients).

Table 3. Simple Correlations for the Full Sample

\begin{tabular}{lrr}
\hline & Self-efficacy & Belonging \\
\hline $\begin{array}{l}\text { Frequency of faculty interaction } \\
\text { in class }\end{array}$ & .20 & .21 \\
$\begin{array}{l}\text { Frequency of faculty interactions } \\
\begin{array}{l}\text { outside of class } \\
\text { Inclusivity }\end{array}\end{array}$ & .12 & -.01 \\
\hline
\end{tabular}

Note. Interpretation of the magnitude of correlations is as follows: $.10-.29$ is a small effect, $.30-.49$ is a medium effect, and .50 or greater is a large effect. Values less than .10 are considered inconsequential (Cohen, 1988).

Primary correlation analyses. Table 4 presents correlations between the introductory course experiences and self-efficacy and belonging separately for first-generation women, continuing generation women, first-generation men, and continuing generation men.

Frequency of faculty interaction in class. First-generation women's self-efficacy and belonging were more strongly related to interactions with faculty inside of class compared to their peers, as evident by medium sized correlations for first-generation women, but small correlations for all other student groups.

Frequency of faculty interaction outside of class. First-generation women's self-efficacy was also more strongly related to interactions with faculty outside of class than their peers. However, interactions with faculty outside of class were not related to students' sense of belonging for any group.
Inclusivity. All students showed a small correlation between perceptions of inclusivity and self-efficacy. However, firstgeneration women's sense of belonging was more strongly correlated with experiences of inclusivity in introductory courses than that of their peers. To the contrary, this relationship was weakest for continuing men, such that there was only a small association between inclusivity and sense of belonging for this group.

\section{LIMITATIONS}

Data for this study were collected at a single time-point. As such, findings from this study cannot be used to infer causation between introductory computing experiences and the outcome variables. Nevertheless, data from this study do suggest that first-generation women with high exposure to select introductory course experiences fare better than first-generation women with low exposure to those experiences

This study also focused on gender and generation status to understand first-generation women's experiences at the intersection of these two identities. A limitation of this work is that it does not consider additional intersectional identities (e.g., race/ethnicity, sexual orientation, SES, religion, etc.). Future work should examine first-generation women's experiences with consideration of their other intersectional identities. Furthermore, because of the infinite number of intersecting identities among women, qualitative or mixed-method research is necessary to further understand the intersectional nature of women's experiences in introductory computing [5].

\section{DISCUSSION}

Results from this study help build an understanding of firstgeneration women's experiences in introductory computing courses. First, we found an intersectional effect for students' sense of belonging and self-efficacy in computing, such that firstgeneration women reported the lowest mean self-efficacy and sense of belonging compared to continuing generation women, first-generation men, and continuing generation men. These group differences are important to reconcile, because both self-efficacy and sense of belonging are key predictors of retention, persistence, and success in the field [3] [4] [8] [19].

In our work, we pinpointed several introductory course characteristics that are linked with first-generation women's sense of belonging and self-efficacy in computing (see Table 4), which we discuss further in the Best Practices section. For now, we wish to draw the reader's attention to a particularly interesting finding: while perceptions of inclusivity were weakly associated with firstgeneration women's self-efficacy $(r=.18)$, perceptions of inclusivity were more strongly associated with first-generation women's sense of belonging $(r=.40)$. This finding speaks to the

Table 4. Simple Correlations by Gender and Generation Status

\begin{tabular}{|c|c|c|c|c|}
\hline & FG Women & Continuing Women & FG Men & Continuing Men \\
\hline \multicolumn{5}{|c|}{ Frequency of faculty interaction in class } \\
\hline Belonging & .42 & .24 & .04 & .16 \\
\hline Self-efficacy & .39 & .12 & .21 & .10 \\
\hline Belonging & .12 & .09 & .03 & -.04 \\
\hline \multicolumn{5}{|l|}{ Inclusivity } \\
\hline
\end{tabular}

Note. Interpretation of the magnitude of correlations is as follows: $.10-.29$ is a small effect, .30 - .49 is a medium effect, and .50 or greater is a large effect; values less than .10 are considered inconsequential (Cohen, 1988). FG = first-generation. 
related, yet distinct nature of self-efficacy and belonging, which has been documented in other research. For instance, while both sense of belonging and self-efficacy are related to academic persistence [3][4], self-efficacy has been shown to be particularly important in predicting leadership behaviors [7] Because women are incredibly underrepresented in leadership roles in computing, and first-generation women report particularly low self-efficacy in our research, it is important to document course experiences conducive to high self-efficacy among women in computing. Our findings suggest that while inclusive environments may not enhance women's self-efficacy in computing, interactions with instructors are positively related to women's self-efficacy in computing. Thus, greater interaction with course instructors may enhance women's self-efficacy, which may in turn increase women's inclination toward leadership within the field.

\subsection{Ancillary Analyses Relevant to Diversity}

First-generation women did not report different introductory course experiences than their peers. However, additional posthoc analyses indicated that women, in the aggregate, reported less frequent interactions with instructors than men, both in the classroom, $F(1,1,999)=20.36 p<.001$, and outside of the classroom, $F(1,1,961)=8.34, p<.01$. Thus, irrespective of generation status, women reported less exposure to instructors. This is troubling, given the association between studentinstructor interactions and computing self-efficacy and belonging for students. Further, the gender gap in exposure to instructor interactions is worrisome in that interactions with instructors may allow students and instructors to develop a rapport that can be to a student's advantage (e.g., the instructor may serve as a mentor, write a letter of recommendation, etc.).

Other analyses indicated that first-generation students are less likely to interact with their instructors in the classroom than continuing generation students, $F(1,1,886)=5.39, p<.05$. This finding is also troubling, given the relationship between interactions with instructors, and students' sense of belonging and self-efficacy (see Table 3) - especially for first-generation women (see Table 4). It may be the case that first-generation students feel less comfortable "in their own skin" in college classrooms than their continuing generation peers, so are relatively less comfortable speaking up in class. On a more positive note, we found that first-generation students are more likely than continuing students to interact with their instructors outside of class (e.g., attending office hours, etc.), $F(1,1,850)=$ $7.30, p<.01$; this activity is associated with enhanced selfefficacy for first-generation students in particular (see Table 4). It is possible that first-generation students may be compensating for low faculty interaction in class with high faculty interaction outside of class. Thus, educators should be aware that some groups of students (e.g., first-generation) may need extra encouragement/incentive to interact in class.

\subsection{Best Practices}

One of the most important takeaways from this study is that even though first-generation women reported the lowest scores on self-efficacy and sense of belonging, some of the introductory course experiences were most strongly associated with the outcomes for this group. Assuming that first-generation women's self-efficacy and sense of belonging are particularly dependent on experiences in introductory courses, our findings present a unique opportunity to broaden participation. In other words, it is possible that relatively low self-efficacy and belonging for first-generation women can be changed, if first- generation women have equal access to introductory course experiences similar to those highlighted in our work. Accordingly, we conclude by suggesting the following best practices for introductory computing course instructors in order to promote high self-efficacy and belonging among all students:

- Increase the frequency of interaction with students in class. This may be implemented via class discussion. Another advantage to class discussion is that it fosters a sense of collaboration in class, which is also known to be beneficial for women in computing [17]. Students may also feel more "connected" to their instructor, if the instructor shares personal stories about their own experiences learning computing concepts.

- Encourage students to drop by during office hours, even if only to say "hello" and introduce themselves. Make clear that many students benefit from some amount of individual attention; this may also be supplemented with recounting personal experiences about asking instructors for help during the faculty member's own college courses.

- Create explicitly inclusive classroom settings. For instance, the use of gender-inclusive language (he or she; him or her) in the classroom is a relatively low-effort means of suggesting to women and men alike that everyone "belongs" in the computing [18].

\section{ACKNOWLEDGMENTS}

CERP is sponsored by two grants from the National Science Foundation awarded to the Computing Research Association: CNS-1246649 and DUE-1431112. BRAID Research is funded by: The Anita Borg Institute (ABI), the National Science Foundation (NSF \#1525737), the Computing Research Association, Facebook, Google, Microsoft, and Intel.

\section{REFERENCES}

[1] Busch, T. 1995. Gender differences in self-efficacy and attitudes toward computers. Journal of Educational Computing Research, 12(2), 147-158.

[2] Cokley, K. 2002. An investigation of academic self-concept and its relationship to academic achievement in African American college students. Journal of Black Psychology, 26(2), 148-164.

[3] Good, C. Rattan, A., \& Dweck, C. S. 2012. Why do women opt out? Sense of belonging and women's representation in mathematics. Journal of Personality and Social Psychology, 102(4), 700-717.

[4] Goodenow, C. 1993. The psychological sense of school membership among adolescents: Scale development and educational correlates. Psychology in the Schools, 30(1), 79-90.

[5] Griffin, K. \& Museus, S. 2011. Using mixed methods to study intersectionality in higher education: New directions for institutional research. Jossey-Bass.

[6] Hurtado, S., Eagan, K., \& Sharkness, J. 2009. Thinking and acting like a scientist: Investigating the outcomes of introductory science and math courses. Association of Institutional Research (AIR) 2009 Paper.

[7] Isaac,C., Kaatz, A., Lee, B., \& Carnes, M. 2012. An educational intervention designed to increase women's leadership self-efficacy. CBE Life Sciences Education, 11(3), 307-322.

[8] Lent, R. W., Brown, S. D., \& Larkin, K. C. 1986. Selfefficacy in the prediction of academic performance and perceived career options. Journal of Counseling Psychology, 33, 265-269. 
[9] Little, T. D., Lindenberger, U., \& Nesselroade, J. R. 1999. On selecting indicators for multivariate measurement and modeling with latent variables: When "good" indicators are bad and "bad" indicators are good. Psychological Methods, 4(2), 191-211.

[10] National Science Foundation. 2015. Women, minorities, and persons with disabilities in science and engineering (No. NSF 15-311). Arlington, VA: National Science Foundation. Retrieved from https://www.nsf.gov/statistics/2015/nsf15311/digest/theme 2.cfm

[11] Pascarella, E. 1980. Student-faculty informal contact and college outcomes. Review of Educational Research, 50(4), 545-595.

[12] Pascarella, E.T., Pierson, C.T., Wolniak G.C., \& Terenzini P.T. 2004. First generation college students: Additional evidence on college experiences and outcomes. The Journal of Higher Education, 75(3), 249-284.

[13] Rosser, S. \& Taylor, M. 2009. Why are we still worried about women in science? Academe: Bulletin of the American Association of University Professors, 95(3) Retrieved from https://www.aaup.org/article/why-are-westill-worried-about-women-science.
[14] Sax, L., Bryant, A., \& Harper, C. 2005. The differential effects of student-faculty interaction on college outcomes for women and men. Journal of College Student Development, 45(6), 642-657.

[15] Stout, J. G. \& Camp, T. 2014. Now what? Action items from social science research to bridge the gender gap in computing research. SIGCAS Computers in Society: Special Issue on Women in Computing, 44, 5-8.

[16] Stout, J. G., Tamer, B. \& Wright, H. M. 2016. The link between feeling welcomed and achievement among first generation students in computing. Proceedings of the AAAS/NSF symposium on Envisioning the Future of Undergraduate STEM Education: Research and Practice.

[17] Stout, J., G., \& Tamer, B. 2016. Collaborative learning eliminates the negative impact of gender stereotypes on women's self-concept. Proceedings for the Annual Meeting of the American Society for Engineering Education.

[18] Stout. J. G., \& Dasgupta, N. (2011). When he doesn't mean you: gender-exclusive language a form of subtle ostracism. Personality and Social Psychology Bulletin, 37(6), 757769.

[19] Walton, M. \& Cohen. G. L. 2007. A question of belonging: race, social fit, and achievement. Journal of Personality and Social psychology, 92(1), 82-96.

\section{Appendix:}

Table A1. Factor Items and Loadings

\begin{tabular}{ll}
\hline Variable & Factor Loading \\
\hline Computing Self-Efficacy & .785 \\
I am confident that I can: find employment in my area of computing interest & .811 \\
I am confident that I can: become a leader in the field of computing & .783 \\
I am confident that I can: win a computing-related contest (e.g., programming contest, robotics contest, & \\
hackathon) & \\
I am confident that I can: get admitted to a graduate computing program & .790 \\
I am confident that I can: complete my undergraduate degree in computing & .783 \\
I am confident that I can: quickly learn a new programming language on your own & .732 \\
I am confident that I can: clearly communicate technical problems and solutions to a range of audiences & .730 \\
Sense of Belonging in Computing & .857 \\
I feel like I belong in computing & .780 \\
I feel like an outsider in the computing community (reverse-coded) & .802 \\
I feel welcomed in the computing community & .859 \\
Inclusivity & \\
Introductory course faculty are inclusive and supportive of women & .844 \\
Introductory course faculty are inclusive and supportive of students of color \\
Introductory course faculty are interested in helping me when I come to them with questions \\
Introductory course faculty are responsive to questions in class \\
Introductory course faculty are responsive to email communication \\
\hline
\end{tabular}

Table A2. Descriptive Statistics for all Variables

\begin{tabular}{lrrrr}
\hline & $\mathrm{N}$ & $\mathrm{Min}$. & Max. & Mean \\
\hline Computing self-efficacy & 2165 & -3.22 & 1.61 & 0.00 \\
Sense of belonging & 2163 & -2.92 & 1.51 & 0.00 \\
Interactions with introductory instructors in class & 2079 & 1.00 & 5.00 & 2.73 \\
Interactions with introductory instructors outside class & 2041 & 1.00 & 5.00 & 1.00 \\
Inclusivity & 2061 & -3.97 & 1.32 & 0.00 \\
\hline
\end{tabular}

Note. $\mathbf{N}$ sizes vary across measures because response to all survey items was voluntary. 